\title{
Mode analysis limitations of ECE-I \& ECE measurements at the plasma edge
}

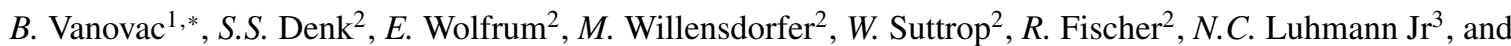 \\ the ASDEX Upgrade Team \\ ${ }^{1}$ DIFFER - Dutch Institute for Fundamental Energy Research, De Zaale 20, 5612 AJ Eindhoven, the Netherlands \\ ${ }^{2}$ Max-Planck-Institut für Plasmaphysik, D-85748 Garching, Germany \\ ${ }^{3}$ Department of Applied Science, the University of California at Davis, Davis, CA 95616, USA
}

\begin{abstract}
Interpretation of the Electron Cyclotron Emission (ECE) and ECE Imaging (ECE-I) measurements concerning the measurement position and the radiation temperature $\left(T_{\mathrm{rad}}\right)$ at the plasma edge is associated with significant uncertainty. Various limitations such as low and high-density limits, relativistic and Doppler shiftbroadening, mode overlap were identified in the past. Here, we analyse the influence of density profile variation onto the radiation temperature in H-mode plasmas at ASDEX Upgrade tokamak. We show that, in the region of steep gradients, the variation of the density profile leads to an outward-shift of the measurement position, towards lower $T_{\text {rad }}$. The analysis is extended towards the study of the contribution of the ordinary mode (Omode) emission to the measured $T_{\text {rad }}$ at the plasma edge. With this analysis, we show the qualitative agreement between experimental observations and modeling when taking into account the O-mode contribution to the ECE measurements. All of these effects are assessed via the Electron Cyclotron Radiation transport forward model that is now routinely used to model the response of the ECE-I and ECE systems in different plasma scenarios.
\end{abstract}

\section{Introduction}

The Electron Cyclotron Emission (ECE) diagnostic is widely used to measure the electron temperature $\left(T_{\mathrm{e}}\right)$ and electron temperature fluctuations $\left(\delta T_{\mathrm{e}}\right)[1]$. The electron cyclotron emission imaging (ECE-I) diagnostic [2] works on the same principle, with the difference that it has multiple lines of sight (LOS), which allow measurements of $\delta T_{\mathrm{e}}$ in a poloidal plane. At ASDEX Upgrade, both diagnostics measure the extraordinary mode (X-mode) microwave intensity, which can be converted into a radiation temperature $T_{\text {rad }}$. The calibrated $T_{\text {rad }}$ can, in many cases, be directly related to $T_{\mathrm{e}}$ at the measuring position defined by the strength of the toroidal magnetic field, only. The measurement position defined in this way is called 'cold' resonance position. However, in specific plasma scenarios and plasma regions, the microwave radiation does not always originate at the cold resonance. This ambiguity is of particular importance for the measurements at the plasma edge. Various types of limitation of the ECE radiometry at the plasma edge such as low and high-density limits, relativistic and Doppler broadening effects are reviewed in [3]. Shine-through in ECE measurements at ASDEX Upgrade is studied in detail in [4,5] and for the DIII-D ECE-I system in [6]. The ECE-I diagnostic at ASDEX Upgrade has an oblique observation angle, and hence an enhanced Doppler shift of the emission frequency. It also suffers from even higher relativistic down-shift of the respective frequencies due to an oblique angle [7] which makes the scrape-off layer (SOL) non-accessible. However, it has been shown that strong perturbations such as density filaments in the SOL can be detected but the reconstruction of an accurate $T_{\mathrm{e}}$ profile from $T_{\mathrm{rad}}$ is, in general, not possible [8]. In [9] the slow variations in density measured with Lithium Beam Emission Spectroscopy (Li-BES) [10] are found to influence the changes in the $T_{\text {rad }}$ signal measured by ECE-I and ECE diagnostics in the pedestal. From here it was concluded that the knowledge of the electron density $\left(n_{\mathrm{e}}\right)$ plays a vital role in the interpretation of the ECE-I in this region. The same effect has been studied for the marginally optically thick plasmas at KSTAR [11]. In this work, we study the response of the ECE-I and ECE systems to the variations of $n_{\mathrm{e}}$ profile in the optically thick $(\tau>3)$ region. To probe the emission volume for each channel and interpret the radiation temperature $T_{\text {rad }}$ we use the electron cyclotron radiation transport model. In this way, we acquire the detailed knowledge on the position of the channels and $T_{\text {rad }}$ associated with it. This information is essential for the interpretation of measured variations in $T_{\text {rad }}$. Since the ordinary mode (O-mode) contribution to the ECE radiation is not perfectly blocked by the polarization filter at the ECE antennas in ASDEX Upgrade, the radiation transport model was extended to take this effect into account. In order to test the modeling of the O-mode contribution, we examine a case where the O-mode contribution to the measured $T_{\text {rad }}$ becomes significant.

In Section 2 we introduce the radiation transport forward model as the primary tool for studies performed in this manuscript. The results on the diagnostics response to the density variation are shown in Section 3 . Furthermore, in

*e-mail: branka.vanovac@ipp.mpg.de 
Section 4 , we show the particular case where the O-mode contribution is significant.

\section{Electron Cyclotron Radiation transport forward model}

At ASDEX Upgrade we routinely use the radiation transport model for interpreting measurements of ECE and ECE-I diagnostics [12, 13]. The radiation transport model accounts for the geometry of the detection systems, and radiation transport effects. For a specific frequency, ray tracing is performed, and the radiation transport equation is solved backwards until the ray reaches the antenna. This model is able to treat both $\mathrm{X}$-mode and O-mode polarization. The radiation transport model delivers the informa-

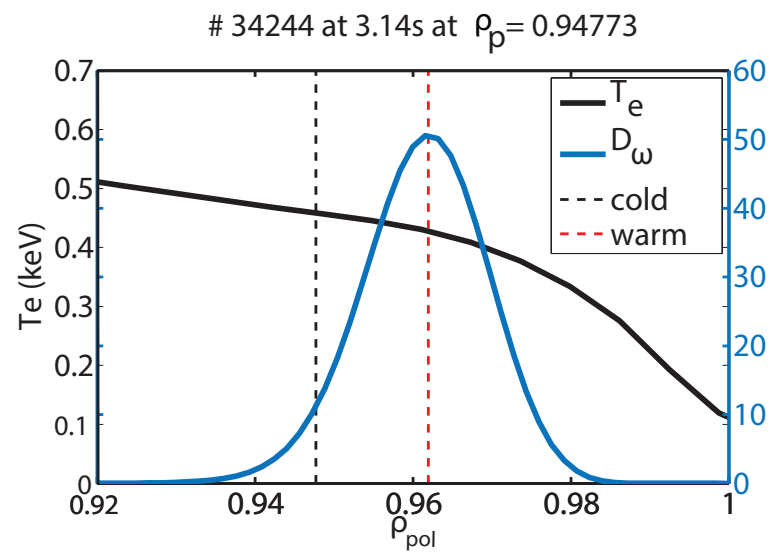

Figure 1. \#34244 at $t=3.14 \mathrm{~s}$ : Midplane edge channel with frequency $\mathrm{f}=110.4 \mathrm{GHz}$ of the ECE-I diagnostic. The cold resonance position at $\rho_{\mathrm{pol}}=0.947$, is determined by the magnetic field strength, and is shown as black dashed line. A birthplace distribution of the observed intensity $\left(D_{\omega}\right)$ is shown in blue. The peak of the $D_{\omega}$ represents a warm resonance of a channel. The $T_{\mathrm{e}}$ profile is shown in a solid black line.

tion on the location of the emitted radiation associated to a certain channel and the $T_{\text {rad }}$ associated with that channel. The location of the emitted radiation is determined as the peak of the birth place distribution of the observed intensity $\left(D_{\omega}\right)$ (see figure 1 and figure 2), defined in [12]. We label this peak as the 'warm' resonance position. This 'warm' resonance can differ from the 'cold' resonance position. The cold resonance $\left(\omega=n e B / m_{e}\right.$, where $\mathrm{n}$ is the harmonic of the cyclotron emission) is determined only by the strength of the magnetic field. The characteristic positions for a single ECE-I midplane channel of frequency $f=110.27 \mathrm{GHz}$ are shown in figure 1. The warm resonance (peak in $D_{\omega}$ ) is shifted outwards in comparison to the cold resonance (black dashed line). The measured electron emission does not originate from a single location but rather from a broad portion of the plasma determined by the width of $D_{\omega}$. The most important broadening effects in the case of the ECE-I system are instrumental and Doppler broadening. The Doppler shift-broadening originates from the oblique observation angle of the ECE-I diagnostics. The outward shift towards lower temperatures

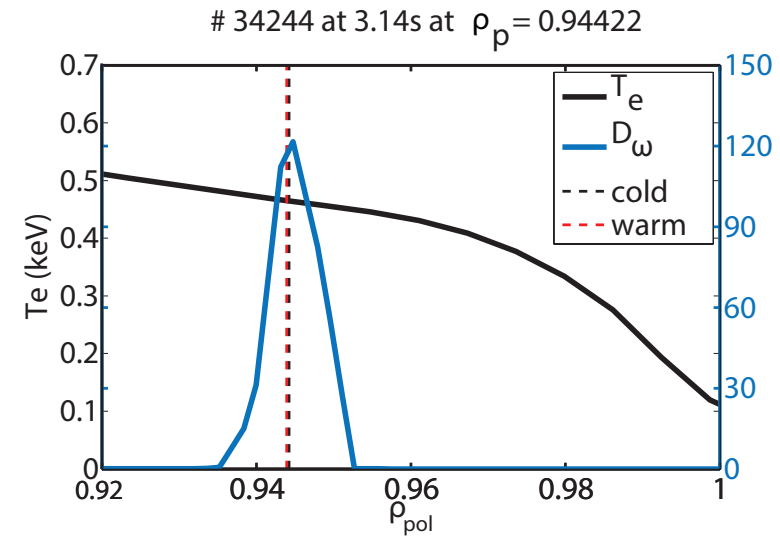

Figure 2. \#34244 at $t=3.14 \mathrm{~s}$ : Midplane edge channel of $\mathrm{f}$ $=110.27 \mathrm{GHz}$ of the ECE diagnostic. The cold resonance position, determined by the magnetic field strength, is shown as black dashed line. A birthplace distribution of the observed intensity $\left(D_{\omega}\right)$ is shown in blue. The peak of the $D_{\omega}$ represents a warm resonance for a respective channel. The $T_{\mathrm{e}}$ profile is shown in a solid black line.

and lower magnetic field compared to the cold resonance (black dashed line) is about $0.5 \mathrm{~cm}$. This is proximately half of the radial resolution for this channel $(1.1 \mathrm{~cm}$ for this channel).

The ECE system, on the other side, has negligible Doppler shift-broadening (see figure 2p as the observation angle is perpendicular to the magnetic field lines. The radial resolution of this ECE channel, determined by the width of $D_{\omega}$, is about $5 \mathrm{~mm}$. However, due to a small divergence of the antenna pattern, a slight Doppler shift can also be observed for the case of the ECE system.

\section{Variation of the density profile}

We apply the radiation transport model to the case of the discharge \# 34244 at $3.14 \mathrm{~s}$. This discharge features strong inter-ELM activity in the low-frequency range measured as $n_{\mathrm{e}}$ and $T_{\mathrm{e}}$ fluctuations simultaneously. To simulate the experiment, a background density profile is modified and used as the input for the Electron Cyclotron Emission Forward Model (ECFM). A modified profile consists of the background profile with $10 \%$ Gaussian perturbation added on top of it. The forward modeling of the electron cyclotron radiation is performed for all channels of both diagnostics. The $T_{\mathrm{e}}$ profile and the equilibrium mapping are kept the same for the two cases. The $n_{\mathrm{e}}$ and $T_{\mathrm{e}}$ profiles are shown in figure 3 The profiles are obtained with Integrated Data Analysis (IDA) within the framework of Bayesian probability theory [14]. The background profile is displayed as a solid black line in figure 3 With added Gaussian perturbation on top of the background profile, we obtain the modulated $n_{\mathrm{e}}$ profile displayed in figure 3 as a black dashed line. The position of the largest change in density profile corresponds to the position of the ECE and ECE-I channels that measure the mode activity in the experiment. In the following, we use the notation as case 1 and case 2 . For case 1 we use the background density 


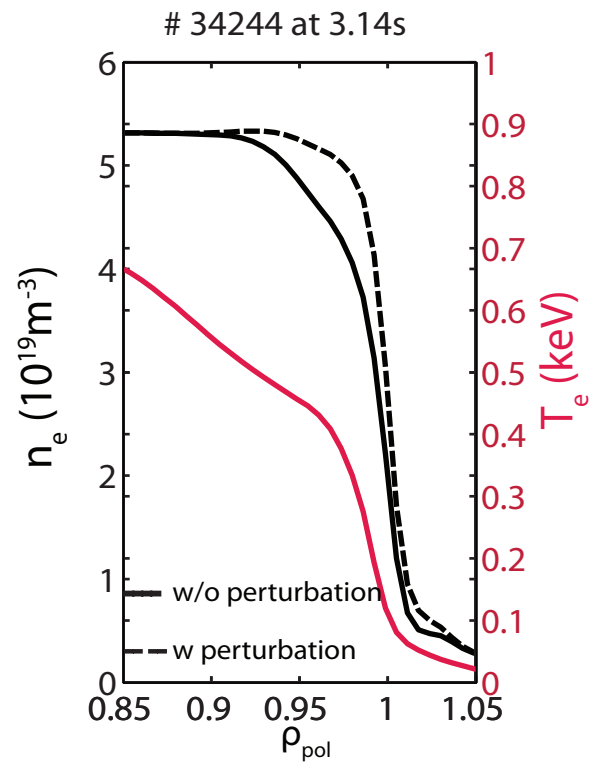

Figure 3. \#34244 at $3.14 \mathrm{~s}$ : Input profiles of $T_{\mathrm{e}}$ (red) and $n_{\mathrm{e}}$ (black) used to model the response of the ECE-I and ECE channels to the presence of the density modulation. Background density profile is shown as a black solid line while the perturbed density profile is displayed as black dashed line. The temperature profile is kept the same for the two cases.

profile (black solid line in figure 3), and for the case 2 we use a density profile with added perturbation (shown as dashed black line in figure 3). Figure 4(a) shows the synthetic $T_{\text {rad }}$ for all channels of the ECE-I system for the two cases shown in figure 3. The channels are mapped onto warm resonances, and they align very well with the profile of the electron temperature inside the confined region. The channels with the cold resonances outside the separatrix obey a relativistic frequency downshift, and are excluded from the analysis. The optical depth $(\tau)$ for the two cases is shown in figure 4(b). The effect of modified density is reflected in the form of a displaced profile of the optical depth (blue profile in figure 4(b)). The background profile is shown in gray. In case 1 the channel position is at $\rho_{\text {pol, } 1}=0.978$. It corresponds to the major radius position of $R_{\mathrm{warm}, 1}=2.138 \mathrm{~m}$. In case 2 , the same channel has its warm resonance at $\rho_{\mathrm{pol}, 2}=0.981$ with $R_{\text {warm }, 2}=$ $2.14 \mathrm{~m}$. The $T_{\text {rad }, 1}=347 \mathrm{eV}$ in case 1 , and $T_{\mathrm{rad}, 2}=323 \mathrm{eV}$ in case 2. For $\Delta R_{\text {warm }}$ of $2 \mathrm{~mm}$ the $\Delta T_{\text {rad }}$ is $\sim 20 \mathrm{eV}$. This makes $3 \%$ change in the $T_{\text {rad }}$ and is comparable to the sensitivity level of the present ECE-I system. The shift of $2 \mathrm{~mm}$ is within the radial resolution of this ECE-I channel $(11.4 \mathrm{~mm})$. The result of the modeling for the profile ECE system is shown in figure 5 For case 1, the position of the channel is $\rho_{\mathrm{pol}, 1}=0.977$ with major radius position at $R_{\text {warm, } 1}=2.135 \mathrm{~m}$. The warm resonance of the same channel for case 2 is $\rho_{\mathrm{pol}, 2}=0.980$ and $R_{\mathrm{warm}, 2}=2.137 \mathrm{~m}$. The $T_{\mathrm{rad}, 1}=350 \mathrm{eV}$, and $T_{\mathrm{rad}, 2}=315 \mathrm{eV}$. The $\Delta R_{\text {warm }}$ is $2 \mathrm{~mm}$, and is the same as for the ECE-I system. However, $\Delta T_{\text {rad }}$ is $35 \mathrm{eV}$ and is higher than that of the ECE-I channel. This makes about $5 \%$ of $T_{\mathrm{e}}$ at the channel position. The radial resolution of this ECE channel is $\sim 6 \mathrm{~mm}$ thus,

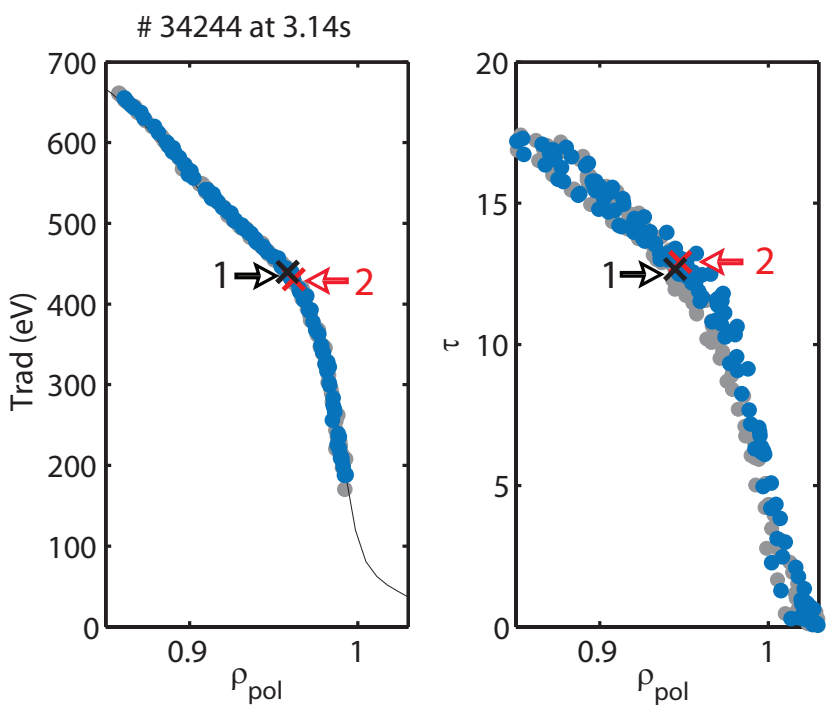

Figure 4. a) Modeled $T_{\text {rad }}$ profile of the ECE-I system shown against warm resonances. The dark gray circles correspond to a case without density perturbation with input density profile shown as a solid black line in figure 3 The blue circles correspond to a case where the perturbed density profile, shown as a dashed black line in figure 3, was used. The channel analyzed in this manuscript is labeled as 1 for the case without perturbation, and 2 with perturbation present. b) Optical depth for ECE-I channels for case 1 and 2, marked as gray and blue circles, respectively.
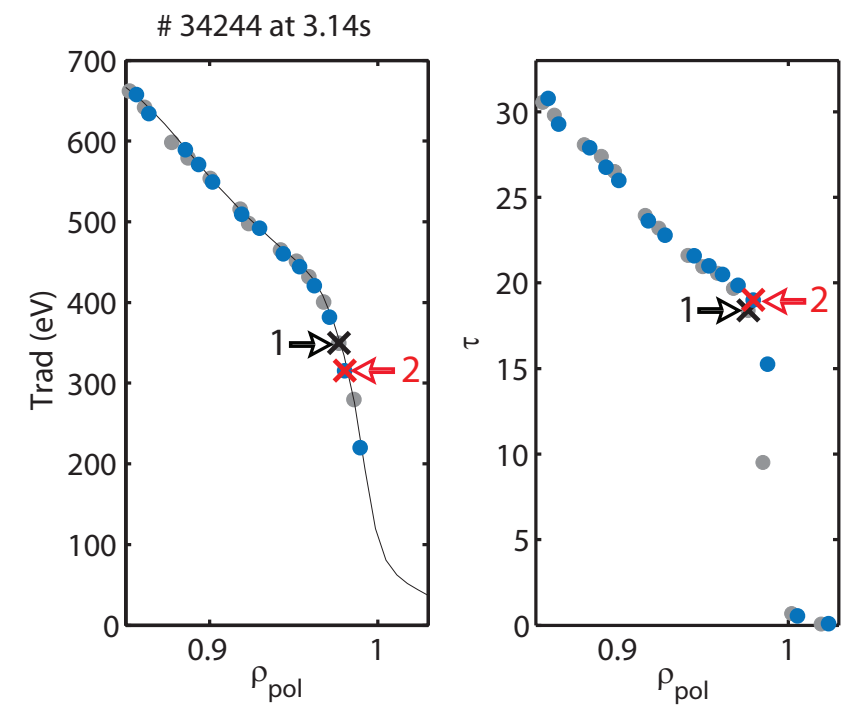

Figure 5. a) Modeled $T_{\text {rad }}$ profile of the ECE system shown against warm resonances of respective channels. The dark gray circles correspond to a case with background density profile (displayed as black solid line in figure 3), and the blue circles correspond to a case with added Gaussian (dashed black profile in figure 3. The channel analyzed in this manuscript is labeled as 1 for the case without perturbation, and 2 with perturbation present. b) Optical depth for ECE channels for case 1 and 2, marked as gray and blue circles, respectively. 
the radial shift of $2 \mathrm{~mm}$ is half of the radial resolution of this diagnostic. Therefore, in both diagnostics, variation of the density profile (no $T_{\mathrm{e}}$ modulation in the modeling) leads to the modulation of measured $T_{\text {rad }}$, visible in a single channel in the optically thick pedestal region. An increase in $n_{\mathrm{e}}$ leads to a decrease of $T_{\mathrm{e}}$, hence if $n_{\mathrm{e}}$ and $T_{\mathrm{e}}$ are modulated in phase, then the ECE underestimates the measured amplitude. Since the density fluctuations during the inter-ELM modes are nearly almost always present, it is necessary to perform the measurements of the density fluctuations simultaneously. As shown, the effect on the channels that should be sensitive to $T_{\mathrm{e}}$ only (the optical depth is high) is non-negligible and falls within the sensitivity of both ECE-I and ECE diagnostics.

\section{O-mode emission}

To measure the X2 (extraordinary polarization $E_{\text {wave }} \perp B_{\mathrm{t}}$, second harmonic) radiation only, it is necessary to prevent the coexistent $\mathrm{O} 2$ (ordinary polarization $E_{\text {wave }} \| B_{\mathrm{t}}$ ) contribution via proper orientation of the polarizing grid. The polarizing grid is not always perfectly aligned (pitch angle changes with plasma safety factor q), therefore some of the O-mode leaks into the waveguides, and some of the $\mathrm{X} 2$ radiation is missing. This way the $\mathrm{O}$-mode contributes to measured radiation temperatures. To study the significance of the O-mode contribution, the radiation transport model described in [12] has been extended. While the formalism is unchanged for $\mathrm{X}$ - and $\mathrm{O}$-mode individually, the calculation of the mixture of $\mathrm{O}$ - and $\mathrm{X}$-mode was added. As the polarizing wire grid is aligned toroidally, the poloidal magnetic field introduces an inherent misalignment of the polarization filter. This misalignment is superimposed by the small toroidal and poloidal inclination of the lines of sight. We calculate the O-mode contribution by projecting the polarization vector $\vec{e}$ of each mode onto the polarization vector of the filter $\vec{p}$. The polarization vector of the microwave is calculated from the cold plasma dispersion relation [15, 16] using the magnetic field vector and the electron density $n_{\mathrm{e}}$ at the separatrix position. This is the common way of calculating the optimal polarization for electron cyclotron resonance heating (ECRH)[17] therefore, also it applies to the ECE case, because of reciprocity. However, it should be noted that the Faraday effect and the Cotton-Mutton effect can change the polarization of the wave while it traverses the SOL[18]. Nevertheless, in this work, any change of wave polarization in the SOL is neglected. Since the intensity depends on the square of the electric field, $T_{\text {rad,mod }}$ is composed of the $\mathrm{X}$ - and O-mode according to:

$$
T_{\text {rad,mod }}=\left(\vec{e}_{\mathrm{X}} \cdot \vec{p}\right)^{2} T_{\text {rad,mod, } \mathrm{X}}+\left(\vec{e}_{\mathrm{O}} \cdot \vec{p}\right)^{2} T_{\text {rad,mod, } \mathrm{O}}
$$

With this formalism synthetic spectra containing a mixture of X-mode and O-mode can be computed. Because the optical depth for the O-mode contribution is usually a lot lower than for X-mode, the birthplace distribution is significantly different and can be much wider for $\mathrm{O}$ mode electron cyclotron emission. This formalism, integrated into the radiation transport model, is applied to one case where the X-mode polarized ECE of the second harmonic is in cut-off for the large fraction of the low-field side (LFS) radius. In figure 6 we show the radial domain of the fundamental $\left(f_{\mathrm{c}}\right)$ and second harmonic $\left(2 f_{\mathrm{c}}\right)$ cyclotron frequency for the magnetic field configuration of this discharge \# 32934 at $t=3.298 \mathrm{~s}$ with an on axis $B_{\mathrm{t}}=-1.8 \mathrm{~T}$. One probing frequency of the ECE radiometer $\left(f_{\mathrm{ECE}}\right)$ is shown as a horizontal solid line. Assumed O-mode ray propagation is depicted in the lower plot. If we were to measure $T_{\text {rad }}$ via the X2 mode it would not be possible due to its cut-off at most of the LFS. Note that $f_{\mathrm{R}}>2 f_{\mathrm{c}}$ for the large fraction of the LFS hence, the X2 cyclotron emission is non-accessible. This includes the cold resonance positions of all ECE measurements in the frequency range $f_{\mathrm{ECE}}=86$ to $98 \mathrm{GHz}$. For clarity, note that the vertical solid black line corresponds to the magnetic axis. Although the X-mode electron cyclotron emission is in cutoff for this discharge, the measured $T_{\text {rad }}$, shown as green circles in figure 7, is significant and is in the range of 200 to $700 \mathrm{eV}$. This intensity originates from the second harmonic O-mode emission, which is not affected by cut-off because $f_{\mathrm{p}}<2 f_{\mathrm{c}}$. Synthetic and measured ECE spec-
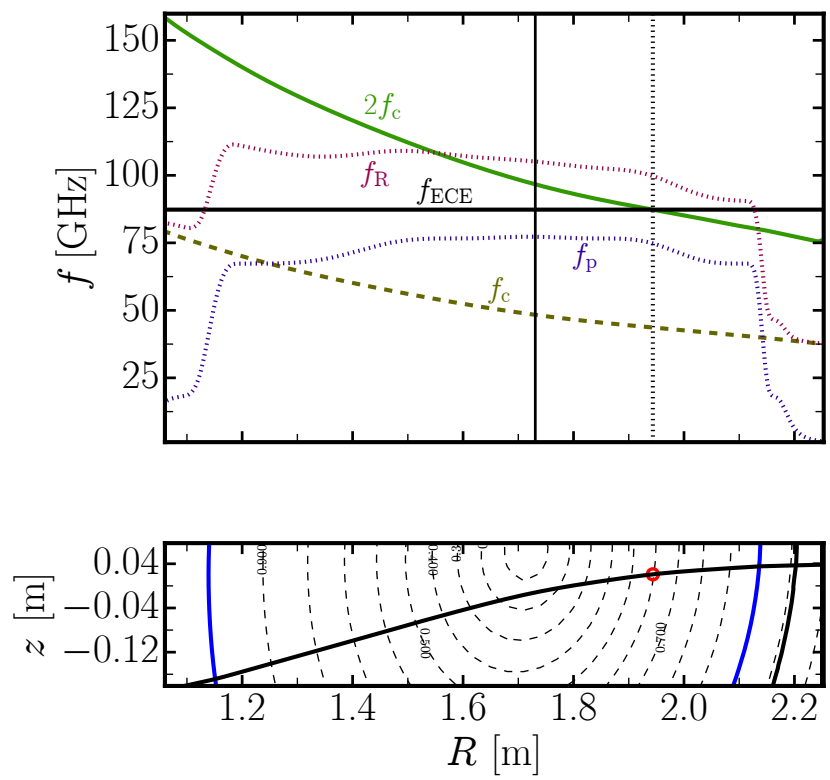

Figure 6. \# 32934 at $t=3.298 \mathrm{~s}$ : Radial dependence of the first and second harmonic of the cyclotron frequency $f_{\mathrm{c}}$, the plasma frequency $f_{\mathrm{p}}$, the right hand cut-off frequency $f_{\mathrm{R}}$ and a measurement frequency of $86 \mathrm{GHz}$. The solid vertical line indicates the position of the magnetic axis. The bottom plot shows the calculated Ordinary mode (O-mode) ray propagation for $f_{\mathrm{ECE}}=$ $86 \mathrm{GHz}$ in the $(\mathrm{R}, \mathrm{z})$ plane.

tra for the discharge \# 32934 at $t=3.298 \mathrm{~s}$ are compared in figure 7 The ECE measurements are shown as green dots and the synthetic ECE spectrum as red triangles. Depicted are $n_{\mathrm{e}}$ and $T_{\mathrm{e}}$-profiles. The kinetic profiles were computed with the integrated data analysis technique[14]. On the same figure, we also show the polarizer efficiency $\left(\vec{e}_{\mathrm{X}} \cdot \vec{p}\right)^{2}$ as purple triangles. The polarizer efficiency is 


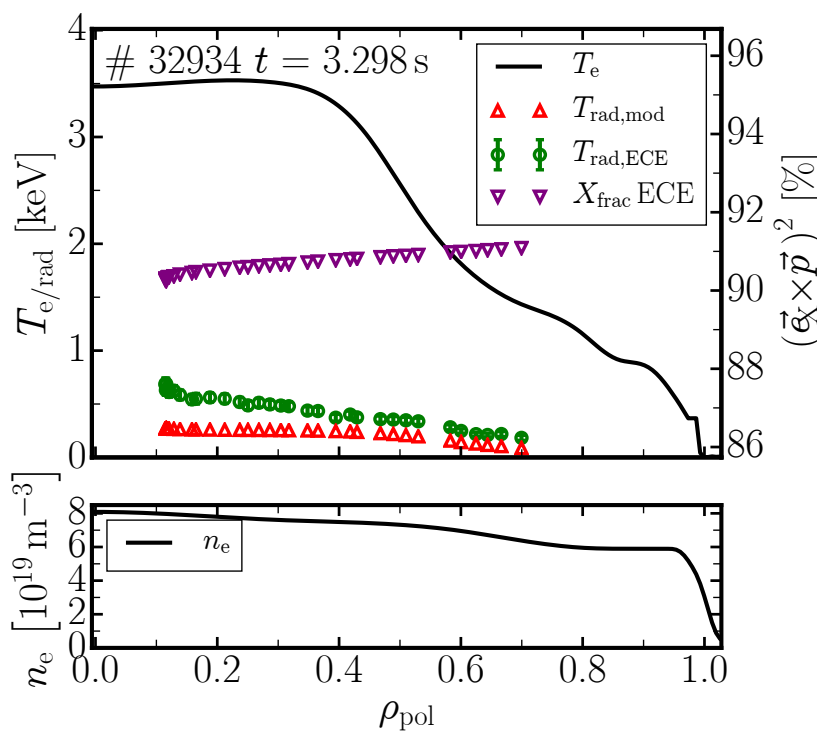

Figure 7. \# 32934 at $t=3.298 \mathrm{~s}: T_{\mathrm{e}}$ (top) and $n_{\mathrm{e}}$ (bottom) profiles versus normalized poloidal radius (black lines). Radiation temperatures $T_{\text {rad,ECE }}$ (measured, green circles) is compared to $T_{\text {rad,mod }}$ (modelled with ECFM, red triangles), with the polarizing efficiency $\left(\vec{e}_{\mathrm{X}} \cdot \vec{p}\right)^{2}$ of $91 \%$ shown as purple triangles.

about $91 \%$. $100 \%$ of polarizing efficiency would correspond to a perfect X-mode polarization. As can be seen, the synthetic $T_{\text {rad,mod }}$ and measured $T_{\text {rad,ECE }}$ do not match perfectly. There are two possible explanations for the observed discrepancy. The first is the influence of wall reflections due to the low optical depth $\tau_{\omega} \approx 0.05$ of the $\mathrm{O} 2$ emission. At the stellarator TJK, full wave calculations were performed to asses which parts of the plasma can contribute to ECE measurements at extremely low optical depth[19]. They found that radiation from any point in the machine is capable of reaching their ECE antenna, because of the wall reflections. The model of infinite reflections between two plane mirrors, which is employed in the radiation transport model [12], is not suitable in this case. Furthermore, the reflections of the metallic surface of the vessel can cause mode conversion between $\mathrm{O}$ - and $\mathrm{X}$-mode. The model assumes that $T_{\text {rad,mod, } \mathrm{X}}=0$ due to the cut-off. It means that any mode conversion from $\mathrm{O}$ to $\mathrm{X}$-mode could greatly increase the modeled $T_{\text {rad }}$ due to the much larger acceptance of X-mode radiation of the polarizing beam splitter. Since only $91 \%$ of the O-mode radiation is rejected in the current model, even small changes in the polarization state of radiation could have a significant impact on the observed $T_{\text {rad }}$. Unlike the influence of wall reflections, this effect is independent on the optical depth and could also affect scenarios in which the O-mode is optically thick. Additional effects, that were omitted in this work, include polarization change in the SOL and Xmode emission reflected from the cut-off layer back to the antenna.

\section{Conclusions}

The radiation transport model enables us to model the response of the ECE-I and ECE systems to different plasma scenarios at the ASDEX Upgrade tokamak. Of special interest are $\mathrm{H}$-mode plasmas with pronounced density fluctuation at the plasma edge region during the inter-ELM periods. We have modeled such a scenario and shown the effect of the density at the pedestal top on an ECE-I and ECE channels both being shifted towards lower magnetic fields and lower radiation temperatures. The effective change in the radiation temperature seen by one channel due to the density is within the sensitivity for both ECE-I and ECE systems and might, therefore, be interpreted as real temperature variation. This effect can have an impact when analyzing the cross phase between $n_{\mathrm{e}}$ and $T_{\mathrm{e}}[20]$. It is not possible to disentangle $n_{\mathrm{e}}$ and $T_{\mathrm{e}}$ fluctuations with ECE-I and ECE measurements only, however, the measurement of $n_{\mathrm{e}}$ fluctuations allows a quantification of their contribution to $T_{\text {rad }}$ via radiation transport model. Furthermore, we have shown that in the particular plasma scenario, where X-mode polarization is in cut-off, the measured radiation temperature is significant. This opens up the possibility to treat the $\mathrm{O}$-mode contribution to the radiation temperatures. However, with the current radiation transport model it is not possible to quantitatively analyze ECE of the second harmonic O-mode measured at ASDEX Upgrade. Nevertheless, it is important to note that even when the X-mode is not in cut-off, $T_{\text {rad }}$ is underestimated by $5-10 \%$ due to O-mode contributions.

\section{Acknowledgments}

This work has been carried out within the framework of the EUROfusion Consortium and has received funding from the Euratom research and training programme 2014-2018 under grant agreement No 633053. The views and opinions expressed herein do not necessarily reflect those of the European Commission.

\section{References}

[1] H.J. Hartfuss, T. Geist, M. Hirsch, Plasma Physics and Controlled Fusion 39, 1693 (1997)

[2] I.G.J. Classen, C.W. Domier, N.C.L. Jr., A.V. Bogomolov, W. Suttrop, J.E. Boom, B.J. Tobias, A.J.H. Donné, Review of Scientific Instruments 85, 11D833 (2014)

[3] W. Suttrop, A. Peeters, IPP report (1996)

[4] S.K. Rathgeber, L. Barrera, T. Eich, R. Fischer, B. Nold, W. Suttrop, M. Willensdorfer, E. Wolfrum, the ASDEX Upgrade Team, Plasma Physics and Controlled Fusion 55, 025004 (2013)

[5] S.S. Denk, R. Fischer, O. Maj, E. Poli, M. Willensdorfer, J. Stober, U. Stroth, W. Suttrop et al., Shinethrough in electron cyclotron emission measurements, in $44^{\text {th }}$ EPS Conference on Plasma Physics (2017), proceedings of the $44^{\text {th }}$ EPS Conference on Plasma Physics 
[6] B.J. Tobias, M.E. Austin, J.E. Boom, K.H. Burrell, I.G.J. Classen, C.W. Domier, N.C.L. Jr., R. Nazikian, P.B. Snyder, Review of Scientific Instruments 83, 10E329 (2012), https://doi.org/10.1063/1.4733742

[7] M. Willensdorfer, S.S. Denk, E. Strumberger, W. Suttrop, B. Vanovac, D. Brida, M. Cavedon, I. Classen, M. Dunne, S. Fietz et al., Plasma Physics and Controlled Fusion 58, 114004 (2016)

[8] B. Vanovac, I. Classen, S. Denk, E. Wolfrum, M. Hoelzl, A. Lessig, F. Orain, N.C.J. Luhmann et al., ELM filaments on ASDEX Upgrade: ECEI observations and modelling, in $43^{\text {th }}$ EPS Conference on Plasma Physics (2016), proceedings of the $43^{\text {th }}$ EPS Conference on Plasma Physics

[9] B. Vanovac, E. Wolfrum, S.S. Denk, F. Mink, F.M. Laggner, G. Birkenmeier, M. Willensdorfer, E. Viezzer, M. Hoelzl, S.J. Freethy et al., Plasma Physics and Controlled Fusion 60, 045002 (2018)

[10] M. Willensdorfer, E. Wolfrum, R. Fischer, J. Schweinzer, M. Sertoli, B. Sieglin, G. Veres, F. Aumayr, the ASDEX Upgrade Team, Review of Scientific Instruments 83, 023501 (2012)

[11] M.K. Ayub, G.S. Yun, W. Lee, H.K. Park, Journal of the Korean Physical Society 70, 268 (2017)

[12] S.S. Denk et al., Radiation transport modelling for the interpretation of oblique ECE measurements, in 19th Joint workshop (EC-19) on Electron Cyclotron Emission (ECE) and Electron cyclotron Resonance Heating (ECRH) (EPJ, 2016)

[13] S.S. Denk, R. Fischer, H.M. Smith, P. Helander, O. Maj, E. Poli, J. Stober, U. Stroth, W. Suttrop, E. Westerhof et al., Plasma Physics and Controlled Fusion 60, 105010 (2018)

[14] R. Fischer, C.J. Fuchs, B. Kurzan, W. Suttrop, E. Wolfrum, the ASDEX Upgrade Team, Fusion Science and Technology 58, 675 (2010)

[15] M. Bornatici, R. Cano, O.D. Barbieri, F. Engelmann, Nuclear Fusion 23, 1153 (1983)

[16] F. Albajar, N. Bertelli, M. Bornatici, F. Engelmann, Plasma Physics and Controlled Fusion 49, 15 (2007)

[17] R. Prater, Physics of Plasmas 11, 2349 (2004), https://doi .org/10.1063/1.1690762

[18] TSUJIMURA et al., Plasma and Fusion Research 1 (2016)

[19] G. Sichardt, E. Holzhauer, A. Köhn, M. Ramisch, T. Hirth, Electron cyclotron emission measurements at the optically thin plasmas of the stellarator TJ-K, in 44th EPS Conference on Plasma Physics (2017)

[20] S.J. Freethy, G.D. Conway, I. Classen, A.J. Creely, T. Happel, A. Köhn, B. Vanovac, A.E. White, Review of Scientific Instruments 87, 11E102 (2016) 